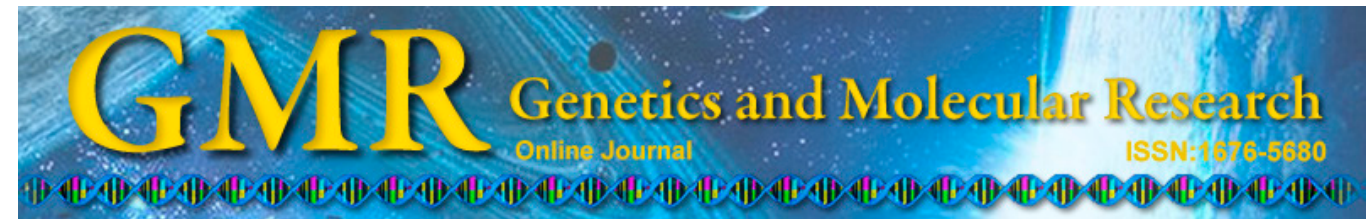

\title{
Optimization of solid-state fermentation conditions for Trichoderma harzianum using an orthogonal test
}

\author{
J.D. Zhang and Q. Yang \\ College of Life Science and Technology, Harbin Institute of Technology, \\ Harbin, China \\ Corresponding author: Q. Yang \\ E-mail: qianycn@163.com
}

Genet. Mol. Res. 14 (1): 1771-1781 (2015)

Received February 21, 2014

Accepted June 26, 2014

Published March 13, 2015

DOI http://dx.doi.org/10.4238/2015.March.13.4

\begin{abstract}
The aim of this study was to develop a protocol for the production of fungal bio-pesticides with high efficiency, low cost, and non-polluting fermentation, while also increasing their survival rate under field conditions. This is the first study to develop biocontrol Trichoderma harzianum transformants $\mathrm{T}_{\mathrm{S} 1}$ that are resistant to benzimidazole fungicides. Agricultural corn stover and wheat bran waste were used as a medium and inducing carbon source for solid fermentation. Spore production was observed, and the method was optimized using single-factor tests with 4 factors at 3 levels in an orthogonal experimental design to determine the optimal culture conditions for $T$. harzianum $\mathrm{T}_{\mathrm{S} 1}$. In this step, we determined the best conditions for fermenting the biocontrol fungi. The optimal culture conditions for $T$. harzianum $\mathrm{T}_{\mathrm{S} 1}$ were cultivated for 8 days, a ratio of straw to wheat bran of 1:3, ammonium persulfate as the nitrogen source, and a water content of $30 \mathrm{~mL}$. Under optimal culture conditions, the sporulation of T. harzianum $\mathrm{T}_{\mathrm{S} 1}$ reached $1.49 \times 10^{10} \mathrm{CFU} / \mathrm{g}$, which was 1.46-fold higher than that achieved before optimization. Increased sporulation of T. harzianum $\mathrm{T}_{\mathrm{S} 1}$ results in better utilization of space and
\end{abstract}


nutrients to achieve control of plant pathogens. This method allows for the recycling of agricultural waste straw.

Key words: Biological pesticides; Conidia; Trichoderma harzianum; Solid-state fermentation

\section{INTRODUCTION}

Trichoderma harzianum $\mathrm{T}_{\mathrm{S} 1}$ belongs to the deuteromycetes Amon, Trichosporon Gang, Trichosporon mesh, sticky spore fungi (Druzhinina et al., 2010) and is widely used as a seed treatment to control disease, promote growth, and increase plant yield (Mastouri et al., 2010; Druzhinina et al., 2010). This fungus releases cell wall-degrading enzymes, which combat plant pathogens through a variety of mechanisms (Ghisalbert and Sivasithamparam, 1991; Monte, 2001; Monteiro et al., 2010). However, T. harzianum $\mathrm{T}_{\mathrm{S} 1}$ has a long culture period and high production costs, which have restricted T. harzianum $\mathrm{T}_{\mathrm{S} 1}$ production levels (da Silva et al., 2012).

Straw and wheat bran are agricultural wastes that are often burnt or abandoned, causing soil fertility to gradually decline as well as the production of fog and haze that cause significant pollution to the environment (Dong et al., 2011). Biodegradation can be used to dispose of straw and wheat bran through the decomposition of straw lignin into small-molecule compounds (Guo et al., 2011) of cellulose or hemicellulose. Therefore, straw can be used to increase soil fertility at a low cost and using methods that do not cause major environmental pollution (Zhang et al., 2011).

In this study, we examined benzimidazole fungicide resistance genes that were transferred into T. harzianum transformants $\mathrm{T}_{\mathrm{S} 1}$ (Li et al., 2008). The aim of this study was to develop a protocol for the production of fungal bio-pesticides with high efficiency, low cost, and non-polluting fermentation, while also increasing the survival rate under field conditions. The carbon sources corn stover and wheat bran waste were used as the medium for the solid fermentation conditions to induce sporulation in T. harzianum $\mathrm{T}_{\mathrm{S} 1}$. Our method reduces the high cost of producing fugal species by using agricultural waste.

\section{MATERIAL AND METHODS}

\section{Fungal strains}

T. harzianum $\mathrm{T}_{\mathrm{S} 1}$ experimental strains were obtained from the Harbin Institute of Technology Life Science and Engineering of China as preserved laboratory strains of T. harzianum $\mathrm{T}_{\mathrm{S} 1}$.

\section{Medium}

The potato dextrose medium (PDA) used contained $200 \mathrm{~g}$ potato, $20 \mathrm{~g}$ sugar, and distilled water to $1 \mathrm{~L}$. The potatoes were washed and peeled, cut into pieces, and immediately placed in boiling distilled water for $30 \mathrm{~min}$. The solution was filtered through gauze. Next, 20 $\mathrm{g}$ sugar and $15 \mathrm{~g}$ agar were mixed and water was added to $1 \mathrm{~L}, 102.9 \mathrm{kPa}$, and then sterilized at $121^{\circ} \mathrm{C}$ for $20 \mathrm{~min}$. For the seed medium, straw and bran were mixed with a grinder and passed through a 60 -mesh sieve; $2.5 \mathrm{~g}$ straw powder and $5 \mathrm{~g}$ wheat bran powder were used. 
The mixture was added to a $250-\mathrm{mL}$ conical flask with $9 \mathrm{~mL}$ distilled water and mixed well. The flask was sealed and sterilized at $121^{\circ} \mathrm{C}$ for $20 \mathrm{~min}$. To optimize the fermentation medium, straw and bran were mixed with a grinder and passed through a 40-mesh sub-sieve, and the powder was used to prepare the medium. The $\mathrm{pH}$ value, inoculum size, and moisture content were optimized for individual experiments, and are described in the relevant sections below.

\section{Inoculation and cultivation}

The same amount of PDA medium was added in a super clean bench to AGAR, cooling, lineation, vaccination, and cultured in a $28^{\circ} \mathrm{C}$ temperature box. Reserved for subsequent fermentation strains. These samples were used for subsequent fermentation.

T. harzianum $\mathrm{T}_{\mathrm{S} 1}$ was grown using the different fermentation medium formulations to identify the optimized production method. Each formulation was tested in triplicate.

A laminar airflow cabinet was used during inoculation. A Petri dish of T. harzianum that had been cultured for more than 5 days was used to select 4-6 areas with densely packed spores. A sterilized scalpel was used to remove a ring, which was added to the sealed flask containing the experimental medium. The inoculated flask was incubated at $28^{\circ} \mathrm{C}$ and shaken daily to ensure that $T$. harzianum $\mathrm{T}_{\mathrm{S} 1}$ had a suitable oxygen supply. After 6 days of growth for the second generation of $T$. harzianum $\mathrm{T}_{\mathrm{S} 1}$, the spores were counted. In the laminar airflow cabinet, $250 \mathrm{~mL}$ was removed from the conical flask containing $T$. harzianum $\mathrm{T}_{\mathrm{S} 1}$ and $1 \mathrm{~g}$ was added to a $50-\mathrm{mL}$ centrifuge tube; $20 \mathrm{~mL}$ water was also added and the mixture was oscillated for $30 \mathrm{~min}$. After standing for $1 \mathrm{~min}, 1 \mathrm{~mL}$ supernatant was added to a test tube and mixed with $9 \mathrm{~mL}$ water. Next, $2 \mu \mathrm{L}$ was added to a $1.5-\mathrm{mL}$ microcentrifuge tube and diluted twice. This process was repeated 3 times for each flask.

\section{Orthogonal design}

An orthogonal test was performed based on the single-factor test according to actual conditions and cost considerations. For T. harzianum field release, we selected incubation time, straw to wheat bran proportion, nitrogen, and water for the 4-factor test with 3 levels of orthogonal experiments to optimize the conditions influencing the composition and to produce the greatest number of spores. Medium production and inoculation using the former method, $\mathrm{L}_{9}\left(3^{4}\right)$ orthogonal design factors, and levels are shown in Table 1 . The experiment was repeated 3 times, and the average was used to determine the best medium composition.

\section{Statistical analysis}

Counts were taken from each centrifuge tube using $1 \mu \mathrm{L}$ diluted broth that was added to the groove of a hemocytometer. After covering, the whole count region was allowed to stand for approximately 1-2 min before counting with a microscope at a magnification of 400X. Three counts were taken from each centrifuge tube.

To calculate the total number of spores in $1 \mathrm{~mL}$, we used the following equation: $(\mathrm{A} / 5)$ x $25 \times 10 \times 10,000 \times \mathrm{B}=50,000 \times \mathrm{A} \times \mathrm{B}(\mathrm{CFU} / \mathrm{mL})$. A represents the total number of spores in 5 squares and $\mathrm{B}$ represents the dilution factor. Here, the counting chamber volume was constant at $0.1 \mathrm{~mm}^{3}$. 


\section{RESULTS}

\section{Effect of proportion of carbon sources on sporulation}

Using the basic medium while other culture conditions remained unchanged, the only change observed was the ratio of straw to wheat bran; the effects of different proportions of carbon on the growth of $T$. harzianum $\mathrm{T}_{\mathrm{S} 1}$ are shown in Figure 1. When straw and bran ratios were $1: 5-1: 2$, the number of spores produced was positively correlated to the ratio between the carbon and straw. When the proportions of straw and wheat bran were $1: 2$, sporulation reached a maximum of $1.02 \times 10^{10} \mathrm{CFU} / \mathrm{g}$, but when the proportion of straw and bran was $3: 4-2: 1$, the number of spores produced was reduced. This may be because the reduced bran, which adds reduced nutrients to the medium, affects the growth and reproduction of T. harzianum $\mathrm{T}_{\mathrm{S} 1}$. Straw not only acts as a carbon source but also plays a supporting role in the medium, creating gaps that enable oxygen to circulate within the medium. Straw and wheat bran have no nutritional value, so these components must be at a reasonable proportion of carbon to provide the best nutrition (Liu et al., 2009) for $T$. harzianum $\mathrm{T}_{\mathrm{S1}}$. Carbon plays a very important role in the growth and metabolism of T. harzianum $\mathrm{T}_{\mathrm{S} 1}$, its cells, and synthesis of the desired product (Ahmed et al., 2009).

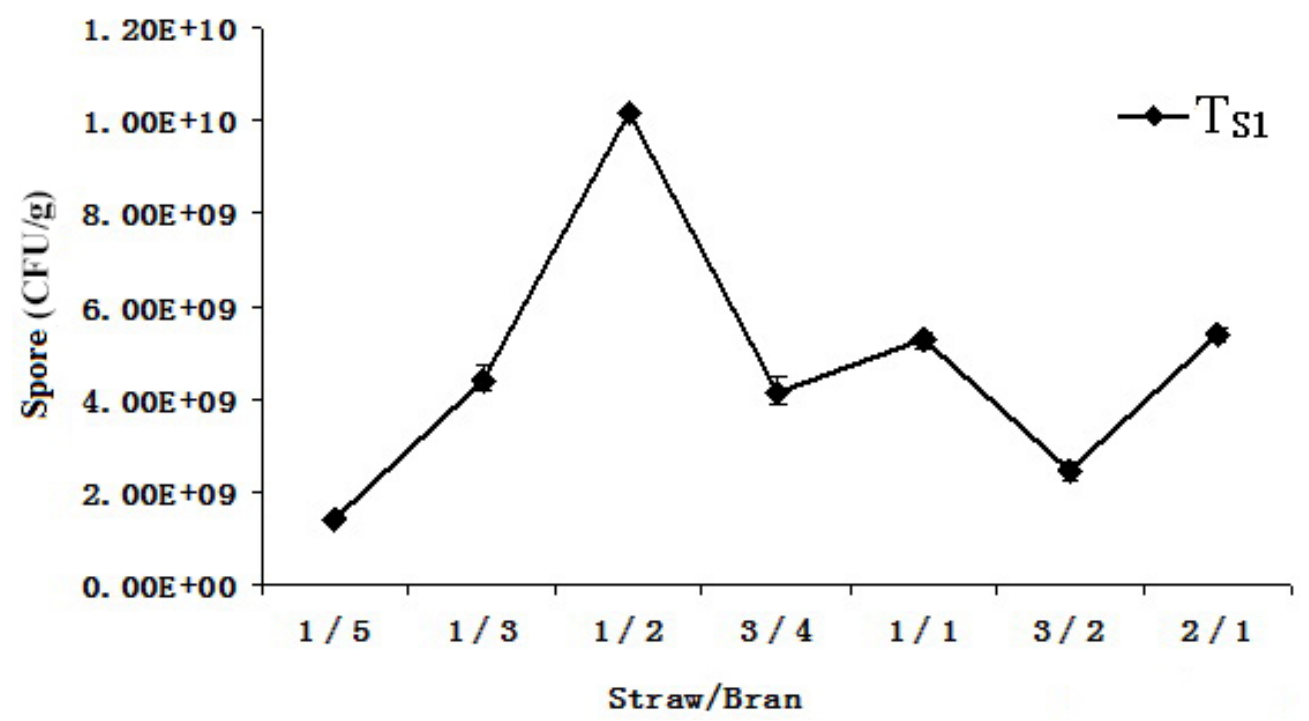

Figure 1. Effect of different proportions of carbon on sporulation.

\section{Impact of nitrogen source on spore production}

Ammonium sulfate, ammonium persulfate, ammonium hydrogen phosphate, ammonium chloride, potassium nitrate, and ammonium nitrate as nitrogen sources were controlled so that the effects of different nitrogen sources on fungal growth could be studied (Figure 2). The effects of different nitrogen sources on the sporulation of T. harzianum $\mathrm{T}_{\mathrm{S} 1}$ showed some differences. In general, growth was better when the 6 nitrogen sources were added compared 
to growth without a nitrogen source; of the 6 nitrogen sources, ammonium persulfate resulted in significantly better $T$. harzianum $\mathrm{T}_{\mathrm{S} 1}$ growth compared to that achieved when using the other 5 sources, reaching $9.29 \times 10^{10} \mathrm{CFU} / \mathrm{g}$. This was followed by ammonium chloride and ammonium sulfate, whereas the lowest amount of growth occurred in the presence of ammonium hydrogen phosphate. After the wheat bran is processed, it can provide some of the nitrogen needed by $T$. harzianum $\mathrm{T}_{\mathrm{S} 1}$, but not all of it, particularly when supporting maximum spore growth. Therefore, additional nitrogen sources are needed.

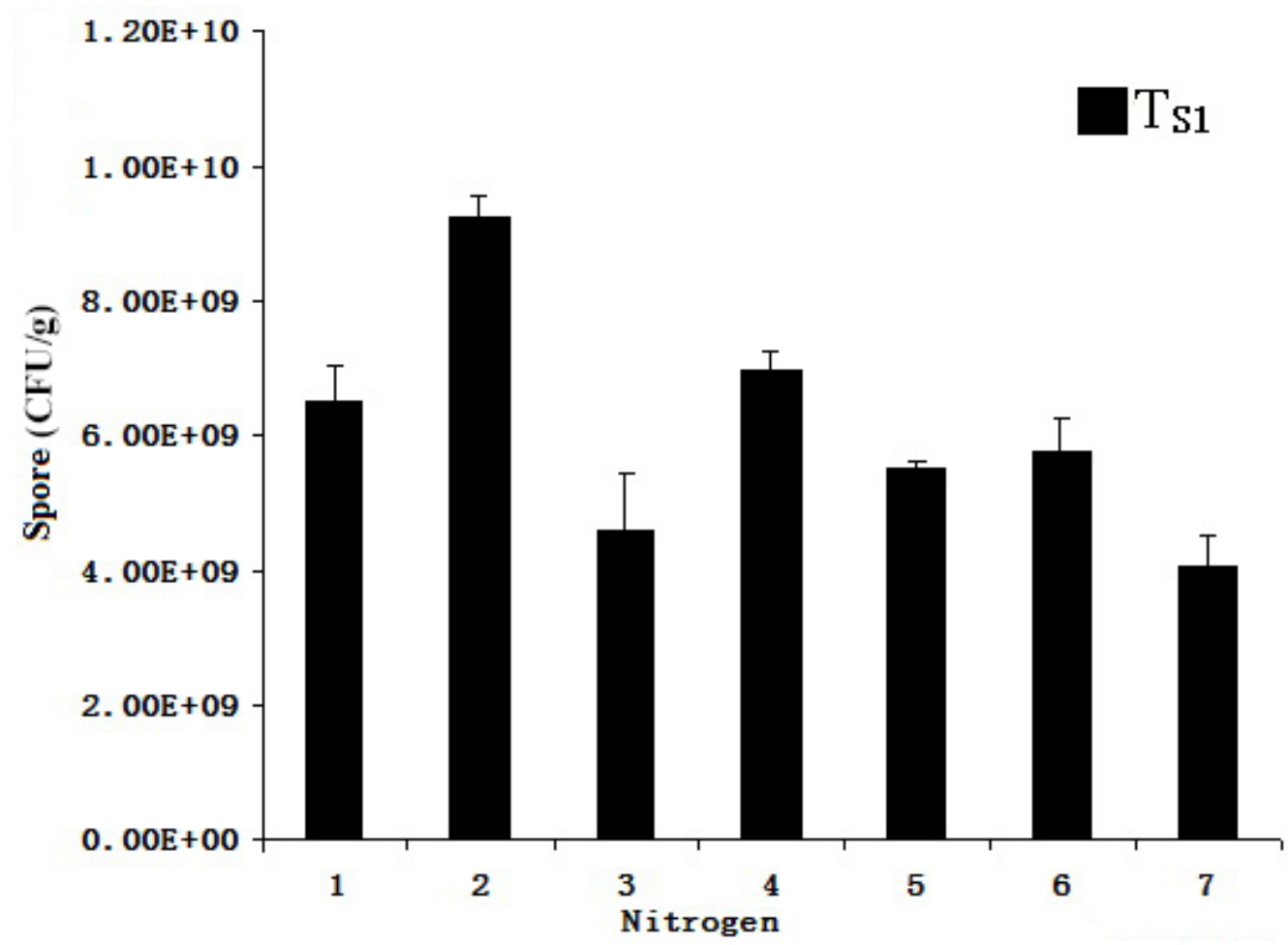

Figure 2. Effect of different nitrogen sources on sporulation. The numbers 1-6 on the horizontal axis correspond to the nitrogen sources ammonium sulfate, ammonium persulfate, ammonium hydrogen phosphate, ammonium chloride, potassium nitrate, and ammonium nitrate, respectively, while 7 is the medium without a nitrogen source.

\section{Effect of moisture content on sporulation}

When the percentage by weight of water is between $60-80 \%$ (Figure 3), the number of spores of $T$. harzianum $\mathrm{T}_{\mathrm{S} 1}$ were positively correlated with the medium's weight percentage of moisture. When water content was $75 \%$, sporulation of T. harzianum $\mathrm{T}_{\mathrm{S} 1}$ reached a maximum of $2.25 \times 10^{9} \mathrm{CFU} / \mathrm{g}$; when the water content was increased to $80 \%$, sporulation decreased. When the moisture content was too high, internal air circulation in the medium was affected, resulting in a lack of oxygen. Therefore, the water content for the culture of T. harzianum $\mathrm{T}_{\mathrm{S} 1}$ should be $75 \%$. 


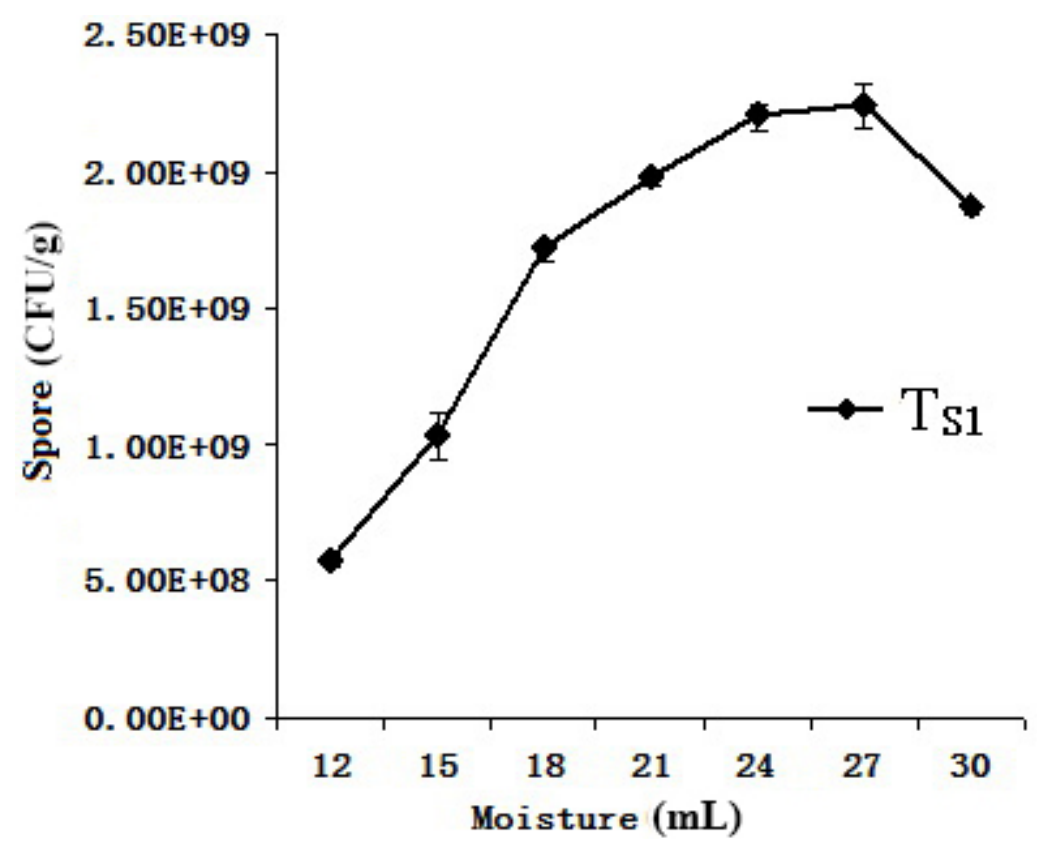

Figure 3. Effect of different water content on sporulation.

\section{Effect of inoculum level on sporulation}

Inoculum levels of $1 \times 10^{3}$ to $1 \times 10^{9} \mathrm{CFU} /$ bottle (Figure 4) were used to compare the number of spores produced, which first increased and then decreased. When the T. harzianum $\mathrm{T}_{\mathrm{S} 1}$ inoculum was $1 \times 10^{7} \mathrm{CFU} /$ bottle, sporulation reached a maximum of $7.61 \times 10^{9} \mathrm{CFU} / \mathrm{g}$. When the inoculation of $1 \times 10^{5} \mathrm{CFU} /$ bottle was changed to $1 \times 10^{6} \mathrm{CFU} /$ bottle, there was a sharp increase in the number of spores, and T. harzianum $\mathrm{T}_{\mathrm{S} 1}$ growth became logarithmic. Lower values for the inoculum, such as $1 \times 10^{3}$ to $1 \times 10^{5} \mathrm{CFU} /$ bottle, were not conducive to T. harzianum $\mathrm{T}_{\mathrm{S} 1}$ spore production in fermentation. When inoculation was $1 \times 10^{8}$ to $1 \times 10^{9}$ CFU/bottle, spores became too concentrated, and spore death occurred because of the toxins produced. This causes hypoxia that affects the survival of other spores, causing an overall decrease in spore number. Therefore, the inoculum for the culture of T. harzianum $\mathrm{T}_{\mathrm{S} 1}$ should be $1 \times 10^{7} \mathrm{CFU} /$ bottle.

\section{Influence of temperature on culture sporulation}

Sporulation experiments were conducted (Figure 5) in the temperature range of $20^{\circ}$ $37^{\circ} \mathrm{C}$, and the results showed that in the temperature range of $20^{\circ}-30^{\circ} \mathrm{C}$, sporulation was positively correlated with temperature. When incubation temperature reached $30^{\circ} \mathrm{C}$, the maximum number of spores was $8.84 \times 10^{9} \mathrm{CFU} / \mathrm{g}$. In the temperature range of $32^{\circ}-37^{\circ} \mathrm{C}$, sporulation was negatively correlated with temperature. The largest number of spores was produced in the temperature range of $28^{\circ}-32^{\circ} \mathrm{C}$. Temperatures below $28^{\circ} \mathrm{C}$ or higher than $32^{\circ} \mathrm{C}$ were not conducive to the germination of spores and their metabolism; when the temperature reached $37^{\circ} \mathrm{C}$, the conidia were killed, drastically reducing the number of spores. 


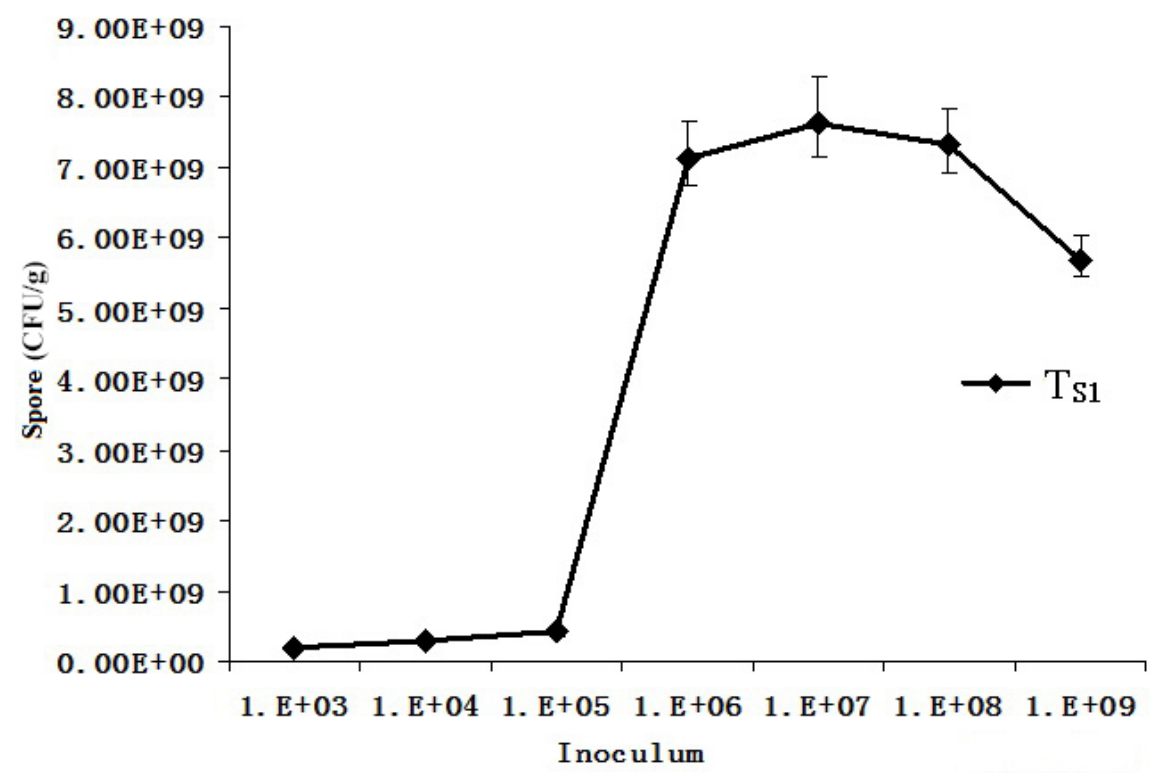

Figure 4. Effect of different inoculum levels on sporulation.

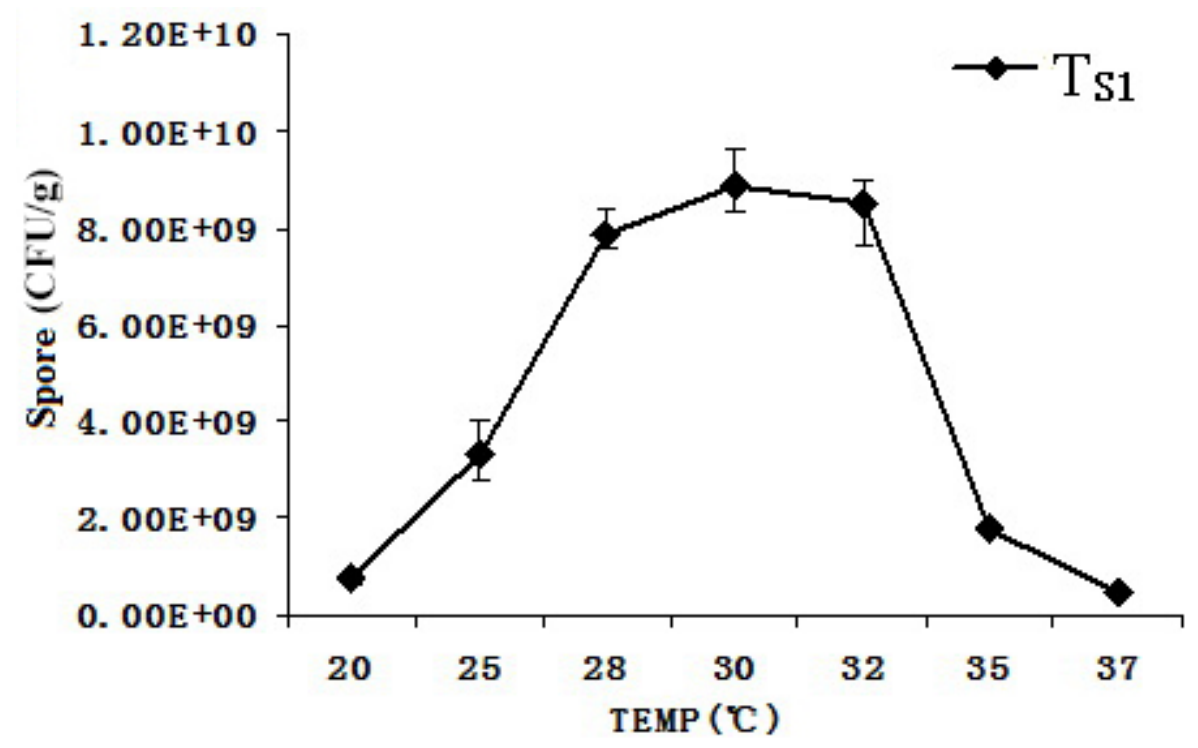

Figure 5. Effect of temperature on sporulation of Trichoderma harzianum $\mathrm{T}_{\mathrm{S} 1}$.

\section{Impact of culture medium pH on sporulation}

The initial $\mathrm{pH}$ value of the medium was changed to examine the effect of $\mathrm{pH}$ on the growth of T. harzianum $\mathrm{T}_{\mathrm{S} 1}$; the results are shown in Figure 6 . When the initial $\mathrm{pH}$ value was between 3 and $9, T$. harzianum $\mathrm{T}_{\mathrm{S} 1}$ generally first increased and then decreased. When the $\mathrm{pH}$ 
value was 6.0,T. harzianum $\mathrm{T}_{\mathrm{S} 1}$ showed maximum sporulation, reaching $8.64 \times 10^{9} \mathrm{CFU} / \mathrm{g}$. A suitable $\mathrm{pH}$ environment for $T$. harzianum $\mathrm{T}_{\mathrm{S} 1}$ enhanced enzyme activity and maintained stability, thereby degrading the straw and wheat bran, enhancing the use of nitrogen, and ultimately producing the largest numbers of spores (Forootanfar et al., 2011).

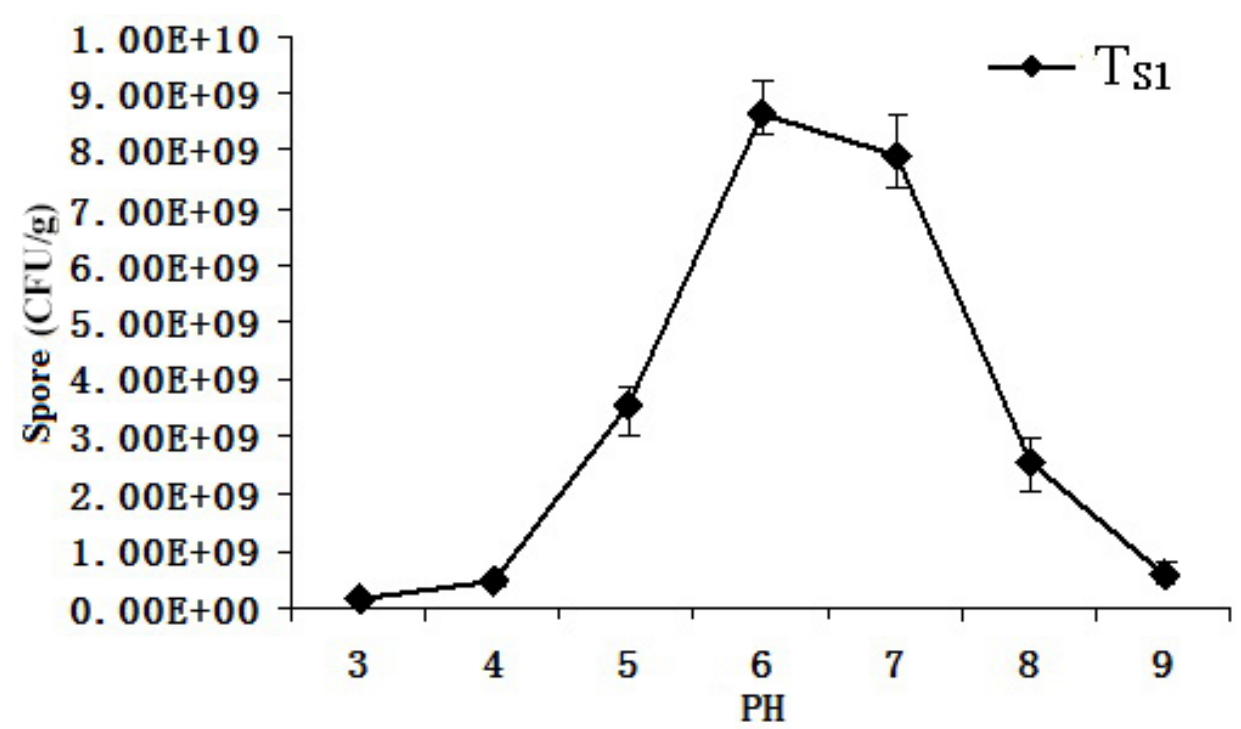

Figure 6. Effect of initial $\mathrm{pH}$ value on sporulation of Trichoderma harzianum $\mathrm{T}_{\mathrm{S} 1}$.

\section{Impact of culture time on sporulation}

When basic medium was used while maintaining all other conditions except culture time, growth of T. harzianum $\mathrm{T}_{\mathrm{S} 1}$ was impacted, as shown in Figure 7. When the number of days of growth increased, the number of $T$. harzianum $\mathrm{T}_{\mathrm{S} 1}$ spores first increased and then decreased. Sporulation of $T$. harzianum $\mathrm{T}_{\mathrm{S} 1}$ showed a smaller increase in the first 5 days and then a logarithmic phase increase until the 9th day. By the 11th day, the maximum number of spores, $1.45 \times 10^{10} \mathrm{CFU} / \mathrm{g}$, was reached. As the length of sporulation time increased, the number of spores decreased, which may be due to spore aging and a decrease in spore viability. For economy and efficiency considerations, incubation time should be 7-9 days for $T$. harzianum $\mathrm{T}_{\mathrm{S} 1}$.

\section{Identification of optimal experimental conditions}

Univariate tests initially identified the best single-factor $T$. harzianum $\mathrm{T}_{\mathrm{S} 1}$ culture conditions; however, optimization by orthogonal combination under multi-factor test conditions was necessary to achieve maximum sporulation. Four factors and 3 levels were used in the orthogonal experiment. T. harzianum $\mathrm{T}_{\mathrm{S} 1}$ was cultured for several different times, while the proportion of straw and wheat bran, nitrogen, and moisture $\mathrm{L}_{9}\left(3^{4}\right)$ were orthogonal design factors (Table 1). The experimental treatment combinations and spore numbers as well as the average and range for each column $(\mathrm{Rj})$ are shown in Table 2. 


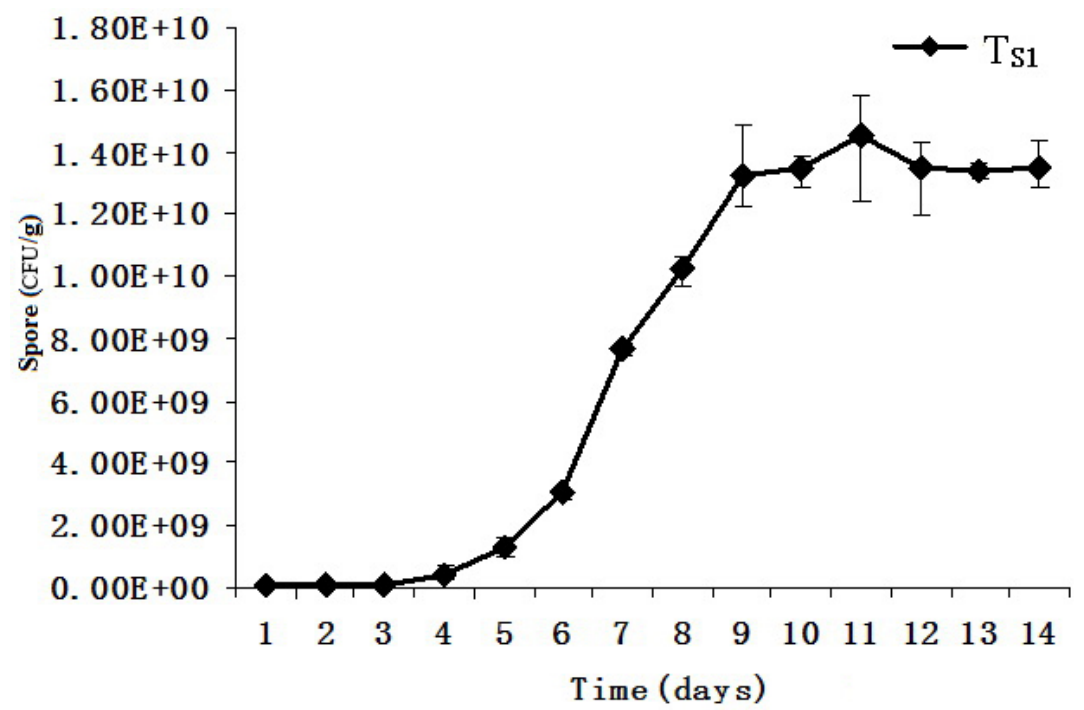

Figure 7. Effect of number of days of culture on sporulation.

Table 1. Trichoderma harzianum $\mathrm{T}_{\mathrm{S} 1}$ cultured for several days with different carbon, nitrogen, and moisture factor levels $\mathrm{L}_{9}\left(3^{4}\right)$ in an orthogonal design test.

\begin{tabular}{lcclc}
\hline Level & \multicolumn{3}{c}{ Factor } \\
\cline { 2 - 5 } & Number (days) & Straw/bran & Nitrogen & Moisture (mL) \\
\hline 1 & 7 & $1 / 3$ & Ammonium sulfate (AS) & 24 \\
2 & 8 & $1 / 2$ & Ammonium persulfate (AP) & 27 \\
3 & 9 & $3 / 4$ & Ammonium chloride (AC) & 30 \\
\hline
\end{tabular}

Table 2. Trichoderma harzianum $\mathrm{T}_{\mathrm{S} 1}$ optimization of solid fermentation conditions using an orthogonal experiment.

\begin{tabular}{|c|c|c|c|c|c|}
\hline \multirow[t]{2}{*}{ Test No. } & \multicolumn{4}{|c|}{ Factor } & \multirow[t]{2}{*}{ Sporulation (CFU/g) } \\
\hline & $\begin{array}{c}\text { A } \\
\text { Number (days) }\end{array}$ & $\begin{array}{c}\text { B } \\
\text { Straw/bran }\end{array}$ & $\begin{array}{c}\text { C } \\
\text { Nitrogen }\end{array}$ & $\begin{array}{c}\mathrm{D} \\
\text { Water content }(\mathrm{mL})\end{array}$ & \\
\hline 1 & 7 & $1 / 3$ & AS & 24 & $1.32 \times 10^{9}$ \\
\hline 2 & 7 & $1 / 2$ & AP & 27 & $1.59 \times 10^{9}$ \\
\hline 3 & 7 & $3 / 4$ & $\mathrm{AC}$ & 30 & $7.40 \times 10^{8}$ \\
\hline 4 & 8 & $1 / 3$ & $\mathrm{AP}$ & 30 & $1.49 \times 10^{10}$ \\
\hline 5 & 8 & $1 / 2$ & $\mathrm{AC}$ & 24 & $7.04 \times 10^{9}$ \\
\hline 6 & 8 & $3 / 4$ & AS & 27 & $1.09 \times 10^{9}$ \\
\hline 7 & 9 & $1 / 3$ & $\mathrm{AC}$ & 27 & $1.00 \times 10^{9}$ \\
\hline 8 & 9 & $1 / 2$ & AS & 30 & $8.47 \times 10^{9}$ \\
\hline 9 & 9 & $3 / 4$ & $\mathrm{AP}$ & 24 & $3.36 \times 10^{9}$ \\
\hline $\mathrm{K} 1$ & $1.21 \times 10^{9}$ & $5.74 \times 10^{9}$ & $3.63 \times 10^{9}$ & $3.90 \times 10^{9}$ & \\
\hline $\mathrm{K} 2$ & $7.67 \times 10^{9}$ & $5.70 \times 10^{9}$ & $6.62 \times 10^{9}$ & $1.23 \times 10^{9}$ & \\
\hline $\mathrm{K} 3$ & $4.27 \times 10^{9}$ & $1.73 \times 10^{9}$ & $2.92 \times 10^{9}$ & $8.04 \times 10^{9}$ & \\
\hline Poor (R) & $6.46 \times 10^{9}$ & $3.97 \times 10^{9}$ & $3.70 \times 10^{9}$ & $6.81 \times 10^{9}$ & \\
\hline Optimization level & A2 & B1 & $\mathrm{C} 2$ & D3 & \\
\hline
\end{tabular}

The experimental results shown in Tables 1 and 2 and the low value of $\mathrm{R}$ reflected the primary and secondary orders of each factor on T. harzianum $\mathrm{T}_{\mathrm{S} 1}$ fermentation as follows: moisture $>$ growth time $>$ straw + bran $>$ nitrogen. The optimal number of growth days was 
8 , the straw and wheat bran ratio was $1: 3$, nitrogen source was ammonium persulfate, and medium moisture content was $30 \mathrm{~mL}$. Under these optimal culture conditions, sporulation of T. harzianum $\mathrm{T}_{\mathrm{S} 1}$ reached $1.49 \times 10^{10} \mathrm{CFU} / \mathrm{g}$, which was 1.46 -fold higher than that obtained before optimization.

\section{DISCUSSION}

The number of spores must reach a particular level before competitive advantages can come into effect and prevent the growth of plant pathogens (Bernal-Vicente et al., 2009; Joshi et al., 2011). The following 6 parameters were optimized using single-factor tests: proportion of straw and wheat bran, $\mathrm{pH}$, nitrogen content, moisture, inoculum level, and growth temperature. We determined the best culture conditions as would occur in field trials and for spore growth. Spores with a competitive advantage will be more effective in controlling plant pathogens.

T. harzianum $\mathrm{T}_{\mathrm{S} 1}$ is effective at killing plant pathogens and acts as a biological pesticide (Gveroska and Ziberoski, 2011), but its application has been limited by its high cost of production and use (Hassan et al., 2009). Agricultural straw waste is typically disposed of by burning, which wastes resources and causes environmental pollution (Qu et al., 2012). The use of straw in the solid-state fermentation of T. harzianum $\mathrm{T}_{\mathrm{S} 1}$ is an effective use of this resource. After the pesticide has been applied to field crops, $T$. harzianum $\mathrm{T}_{\mathrm{S} 1}$ can be applied as a pesticide and will continue to develop and grow as other pathogens are killed. T. harzianum $\mathrm{T}_{\mathrm{S} 1}$, based on the original medium, is easy to breed and has a higher survival rate, and straw fermentation can provide a variety of plants with NPK fertilizer (Liu et al., 2009), as well as promote plant growth.

To make the fungal degradation of straw more thorough and easy to conduct during solid-state fermentation, the straw can be pretreated to break the cellulose solid structure. Various physical or chemical treatments can be used, such as steam explosion, microwave, and acid treatment. Further studies are required to examine the pretreatment of straw before fermentation so that during the fermentation process, it releases larger amounts of beneficial trace elements that can be used as biological pesticides and fertilizers.

\section{CONCLUSIONS}

As revealed by single-factor experiments, the optimal culture conditions for T. harzianum $\mathrm{T}_{\mathrm{S} 1}$ were a straw to bran ratio of $1: 2, \mathrm{pH}$ value of 6.0 , nitrogen source of ammonium persulfate, water content of $75 \%$, inoculation of $1 \times 10^{7}$ cells/bottle, and a growth temperature of $30^{\circ} \mathrm{C}$. Further, by examining 4 factors and 3 levels in an orthogonal experiment, the culture conditions for T. harzianum $\mathrm{T}_{\mathrm{S} 1}$ were identified as growth for 8 days, straw to bran ratio of 1:3, nitrogen source of ammonium persulfate, and moisture of $30 \mathrm{~mL}$. Under these optimal culture conditions, sporulation of T. harzianum $\mathrm{T}_{\mathrm{S} 1}$ reached $1.49 \times 10^{10} \mathrm{CFU} / \mathrm{g}$, which was 1.46 -fold higher than the level obtained before optimization. Trichoderma harzianum $\mathrm{T}_{\mathrm{S} 1}$ can use straw and wheat bran as a carbon source for growth on a solid medium, resulting in good sporulation, which can be maintained for up to 10 days and is beneficial for its widespread use as a biological pesticide.

\section{REFERENCES}

Ahmed S, Bashir A, Saleem H and Saadia M (2009). Production and purification of cellulose-degrading enzymes from a filamentous fungus Trichoderma harzianum. Pak. J. Bot. 41: 1411-1419. 
Bernal-Vicente A, Ros M and Pascua JA (2009). Increased effectiveness of the Trichoderma harzianum isolate T-78 against Fusarium wilt on melon plants under nursery conditions. J. Sci. Food Agric. 89: 827-833.

da Silva LC, Honorato TL, Cavalcante RS, Franco TT, et al. (2012). Effect of pH and temperature on enzyme activity of chitosanase produced under solid stated fermentation by Trichoderma spp. Indian J. Microbiol. 52: 60-65.

Dong YF, Lu J, Jin H and Yang RF (2011). Comparison of enzymatic hydrolysis of leaves, husks and pith of corn stalk. Fut. Mat. Eng. Ind. Appl. 365: 240-244.

Druzhinina IS, Kubicek CP, Komon-Zelazowska M, Mulaw TB, et al. (2010). The Trichoderma harzianum demon: complex speciation history resulting in coexistence of hypothetical biological species, recent agamospecies and numerous relict lineages. BMC Evol. Biol. 10: 94.

Forootanfar H, Faramarzi MA, Shahverdi AR and Yazdi MT (2011). Purification and biochemical characterization of extracellular laccase from the ascomycete Paraconiothyrium variabile. Bioresour. Technol. 102: 1808-1814.

Ghisalbert EL and Sivasithamparam K (1991). Antifungal antibiotics produced by Trichoderma spp. Soil Biol. Biochem. 23: 1011-1020.

Guo P, Mochidzuki K, Cheng W, Zhou M, et al. (2011). Effects of different pretreatment strategies on corn stalk acidogenic fermentation using a microbial consortium. Bioresour. Technol. 102: 7526-7531.

Gveroska B and Ziberoski J (2011). The influence of Trichoderma harzianum on reducing root rot disease in tobacco seedlings caused by Rhizoctonia solani. Int. J. Pure. Appl. Sci. Technol. 2: 1-11.

Hassan F, Meens J, Jacobsen HJ and Kiesecker H (2009). A family 19 chitinase (Chit30) from Streptomyces olivaceoviridis ATCC 11238 expressed in transgenic pea affects the development of T. harzianum in vitro. J. Biotechnol. 143: 302308.

Joshi BB, Singh V, Srivastava SN and Jilal R (2011). Biological control of red rot disease of sugarcane through Trichoderma harzianum and Trichoderma viride. Indian Phytopathol. 4: 2248-2249.

Li M, Yang Q and You ZQ (2008). Neurospora crassa BenR gene point conversion in the harzianum. J. Southwest China Normal Univ. 4: 58-63.

Liu TX, Liu YQ and Ma JL (2009). Study on fermentation of corn straw powder with multi-strains in feed production of proteins. Anim. Husbandry Feed Sci. 9: 36-38.

Mastouri F, Björkman T and Harman GE (2010). Seed treatment with Trichoderma harzianum alleviates biotic, abiotic, and physiological stresses in germinating seeds and seedlings. Phytopathology 100: 1213-1221.

Monte E (2001). Understanding Trichoderma: between biotechnology and microbial ecology. Int. Microbiol. 4: 1-4.

Monteiro VN, do Nascimento SR, Steindorff AS, Costa FT, et al. (2010). New insights in Trichoderma harzianum antagonism of fungal plant pathogens by secreted protein analysis. Curr. Microbiol. 61: 298-305.

Qu C, Li B, Wu H and Giesy JP (2012). Controlling air pollution from straw burning in China calls for efficient recycling. Environ. Sci. Technol. 46: 7934-7936.

Zhang J, Zeng G, Chen Y, Yu M, et al. (2011). Effects of physico-chemical parameters on the bacterial and fungal communities during agricultural waste composting. Bioresour. Technol. 102: 2950-2956. 